\title{
Improving underwater imaging in an amplitude modulated laser system with radio frequency control technique
}

\author{
L. De Dominicis \\ luigi.dedominicis@enea.it
}

\section{Ferri de Collibus \\ mario.ferridecollibus@enea.it}

\section{G. Fornetti}

giorgio.fornetti@enea.it

\section{Guarneri}

massimiliano.guarneri@enea.it

\section{Nuvoli}

marcello.nuvoli@enea.it

\section{R. Ricci}

roberto.ricci@enea.it

\section{Francucci}

massimo.francucci@enea.it

\begin{abstract}
ENEA - Italian National Agency for New Technologies, Energy and Sustainable Economic Development, Via. E. Fermi 45, C.P.65, I-00044 Frascati

ENEA - Italian National Agency for New Technologies, Energy and Sustainable Economic Development, Via. E. Fermi 45, C.P.65, I-00044 Frascati

ENEA - Italian National Agency for New Technologies, Energy and Sustainable Economic Development, Via. E. Fermi 45, C.P.65, I-00044 Frascati

ENEA - Italian National Agency for New Technologies, Energy and Sustainable Economic Development, Via. E. Fermi 45, C.P.65, I-00044 Frascati

ENEA - Italian National Agency for New Technologies, Energy and Sustainable Economic Development, Via. E. Fermi 45, C.P.65, I-00044 Frascati

ENEA - Italian National Agency for New Technologies, Energy and Sustainable Economic Development, Via. E. Fermi 45, C.P.65, I-00044 Frascati

ENEA - Italian National Agency for New Technologies, Energy and Sustainable Economic Development, Via. E. Fermi 45, C.P.65, I-00044 Frascati
\end{abstract}

We present the results of an experiment aimed to demonstrate the low-pass filter dependence of water backscattered power in an amplitude-modulated laser scanner for underwater 3D imaging. We also demonstrate that improvements in target imaging are obtained by allowing the device to operate in the stop-band region. A simple model is described to account for the physics underlying the effect and suggesting future experimental schemes based on demodulated detection techniques. [D0I: 10.2971/jeos.2010.10004]

Keywords: underwater imaging, amplitude-modulated laser, optical noise, water scattering, low-pass filter

\section{INTRODUCTION}

The development of laser scanners for three-dimensional (3D) imaging of targets in submarine environment is a research area of interest for several potential users. These include, for instance, offshore oil companies for pipelines and platforms inspection, military institutions for target recognition and governmental organizations for survey of the submarine archaeological heritage. The interest stems from the potentiality of laser devices of visualizing farther than conventional cameras [1]. Up to a decade ago, laser imagers based on amplitude-modulated (AM) lasers at radio frequency (RF) had been receiving less attention than systems operating with pulsed or non-modulated CW laser sources [2], despite the fact that $3 \mathrm{D}$ target imaging in an AM device relies on the measurement of the signal phase and does not require an a priori estimation of target distance, or even a dual laser illumination. Nevertheless, the recent advent on the market of diode lasers in the blue spectral region with power up to $100 \mathrm{~mW}$, and the possibility of analogue modulation up to $350 \mathrm{MHz}$ without the need of any electro-optical stage, have boosted the interest towards AM systems, mainly in view of the possibility of engineering compact devices [3].

As for all optical underwater imaging systems, the performances of an AM device depend on the efficiency of the solutions adopted to reject the optical noise originating from pho- tons backscattered by water. In this respect, the possibility of optical noise rejection based on RF filtering characterizes AM systems. The first experimental demonstration of the existence of a cut-off frequency in the RF range due to the low-pass filter frequency response of the ocean water backscattering for a 100 ps laser pulse excitation dates back to 2000 [4], following a theoretical analysis of L. Mullen et al. [5]. Improvements in underwater target contrast were soon after demonstrated by modulating the pulse's intensity above the cut-off value [6]. However, an experimental demonstration of the low-pass frequency response of water backscattering for continuous AM laser excitation is still lacking [7]. Beside the relevance for validating theoretical models inspired by previous studies and overhauled to better simulate our device performances [8, 9], these experimental data could give insights on how instrumental, rather than physical parameters, shape the filter function.

In the present paper we report the results of an experiment aimed at measuring the frequency response of water backscattering as recorded by an AM device based on a diode laser emitting radiation at $\lambda=405 \mathrm{~nm}$ and propagating in a $25 \mathrm{~m}$ long test tank filled with water of varying turbidity. In addition, we also demonstrate that better performances are obtained when the device is operating in the stop-band fre- 
quency region, by recording the linear profile of a simple target immersed at $3.7 \mathrm{~m}$ from the entrance window of the tank.

The paper is organized as follows: in Section 2 a simple theoretical approach is used to illustrate the physics underlying the low-pass filter behavior of water backscattering under AM excitation, in Section 3 the experimental apparatus is described and in Section 4 the results of experimental measurements reported.

\section{BASIC THEORY}

In an AM laser 3D imager the intensity of a spatially collimated laser beam is sinusoidally modulated at RF. The range of the target region illuminated by the laser is determined by measuring the phase shift, with respect to a reference signal, of the reflected radiation. A 3D image can be recorded by sweeping the laser onto the target's whole surface. The reflected radiation is collected by a lens of radius $r_{0}$, mounted in a bistatic geometry where $r_{r e c}$ is the laser-receiver separation. The whole receiving optics is characterized by an angular field of view (FOV) $\theta_{\text {fov }}$. If the target is immersed in water, a considerable degradation on the image quality comes from the optical noise due to photons backscattered by water and falling into the receiver FOV. To get rid of this unwanted contribution in AM systems, it is possible to exploit the low-pass filter dependence of water backscattered power on the modulating frequency.

A rigorous approach to demonstrate this property, within the framework of the radiative transfer theory (RTT), consists in solving an integro-differential Radiative Transfer Equation (RTE) which accounts for both device parameters and water optical properties. A method for finding particular solutions of the problem can be found in [9]. Here we adopt the simplest model by restricting attention to an ideal system, because the complexity of the calculations could otherwise overshadow the basic physics underlying the process. We consider a monostatic $\left(r_{r e c}=0\right)$ system with infinitesimally narrow $\theta_{\text {fov }}$, so that only perfectly backscattered photons are detected. A perfectly collimated AM laser source is located at the plane $z=0$, the separation interface between air $(z<0)$ and water $(z>0)$. The laser is turned on at time $t=0$ and emits photons along the positive $z$ axis. The RF modulation causes the number of photons $N_{l}(z, t)$ to propagate as a transverse wave with a wave-vector $k_{l}=2 \pi n f_{m} / c$, where $f_{m}$ is the RF modulation frequency, $n$ the water refractive index and $c$ the speed of light in vacuum. It turns out that at $t>0$ in the spatial point $z>0$ we have

$$
\begin{aligned}
N_{l}(z, t) & =N_{0} e^{-k z}\left\{1+m \cos \left[k_{l} z-2 \pi f_{m} t\right]\right\} \\
& =N_{0} e^{-k z}\left\{1+m \cos \left[2 \pi f_{m}\left(\frac{z n}{c}-t\right)\right]\right\}
\end{aligned}
$$

where $k$ is the water attenuation coefficient and the exponential factor accounts for attenuation due to photons that are absorbed and scattered off the laser path. Moreover, $m \in[0,1]$ is the modulation index and $N_{0}$ is the number of photons at $z$ and $t$ for the laser operating in the c.w. mode $(m=0)$. If $\beta$ is the backward scattering coefficient and we restrict the analysis to single backscattering events, the number $N_{b}(t)$ of backscat-

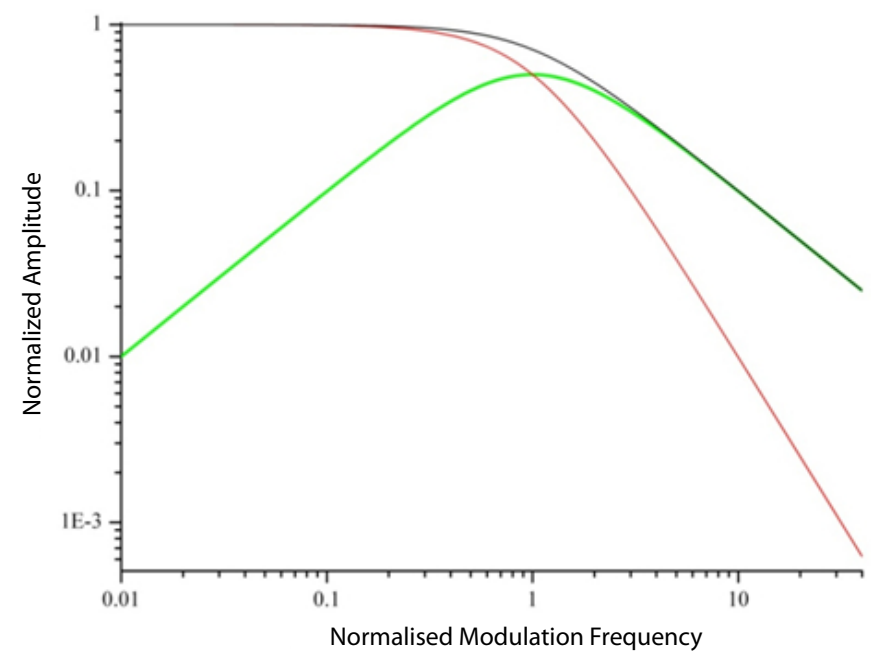

FIG. 1 Normalized optical noise vs $f_{m} / f_{c}$. Black line: amplitude of the modulated component. Red line: amplitude of the in-phase component. Green line: amplitude of the quadrature component.

tered photons at time $t$ on the detector is obtained by integrating over $z$ the function $\beta N_{l}(z, t-z n / c)$ which gives the number of backscattered photons at $z$ at the instant $t-z n / c$, where $z n / c$ accounts for the travelling time from $z$ to the detector. The exponential decay factor allows, at first approximation, to extend the integral up to $z=\infty$, thus giving

$$
\begin{aligned}
N_{b}(t) \approx \beta N_{0} \int_{0}^{\infty} e^{-2 k z}\left\{1+m \cos \left[2 \pi f_{m}\left(\frac{2 z n}{c}-t\right)\right]\right\} d z \\
=\frac{\beta N_{0}}{2 k}\left[1+\frac{m}{1+\left(f_{m} / f_{c}\right)^{2}} \cos \left(2 \pi f_{m} t\right)\right. \\
\left.+m \frac{f_{m} / f_{c}}{1+\left(f_{m} / f_{c}\right)^{2}} \sin \left(2 \pi f_{m} t\right)\right] \\
=\frac{\beta N_{0}}{2 k}\left[1+\frac{m}{\sqrt{1+\left(f_{m} / f_{c}\right)^{2}}} \cos \left(2 \pi f_{m} t+\phi\right)\right]
\end{aligned}
$$

where the cut-off frequency $f_{c}=k c / 2 \pi n$ has been introduced and $\phi=\tan ^{-1}\left(-f_{m} / f_{c}\right)$ The modulated component of the backscattered photons flow follows the laser's intensity modulation with an amplitude and a phase delay depending on frequency. Amplitude depends on $f_{m}$ as a first order Butterworth lowpass filter while the in-phase and quadrature components behave as a first order lowpass filter and a pass-band filter respectively. The functions are plotted in Figure 1 versus $f_{m}$ in units of $f_{c}$ and for constant $m$.

This simple calculation retains the result in [9] for the ideal system here considered, giving a cut-off frequency which depends in this case only on the attenuation coefficient and on the refractive index, that is on intrinsic properties of the medium. Obviously, the mean number of photons backscattered over a modulation period does not depend on the frequency and is given by $\beta N_{0} / 2 k$. As far as only single backscattering events are considered, the inphase and quadrature decomposition shows that for modulation frequencies less than the single scattering rate $f_{c}$ the backscattered power is driven coherently by the AM laser. Coherence is gradually lost as the modulation frequency becomes comparable to $f_{c}$ The modu- 


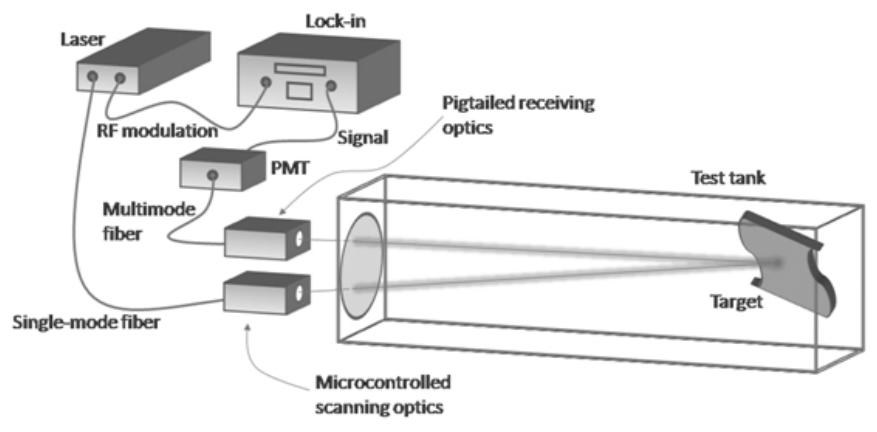

FIG. 2 Experimental apparatus. The scheme is not to scale. In the ranging experiment the target was located at $3.7 \mathrm{~m}$ from the optical window.

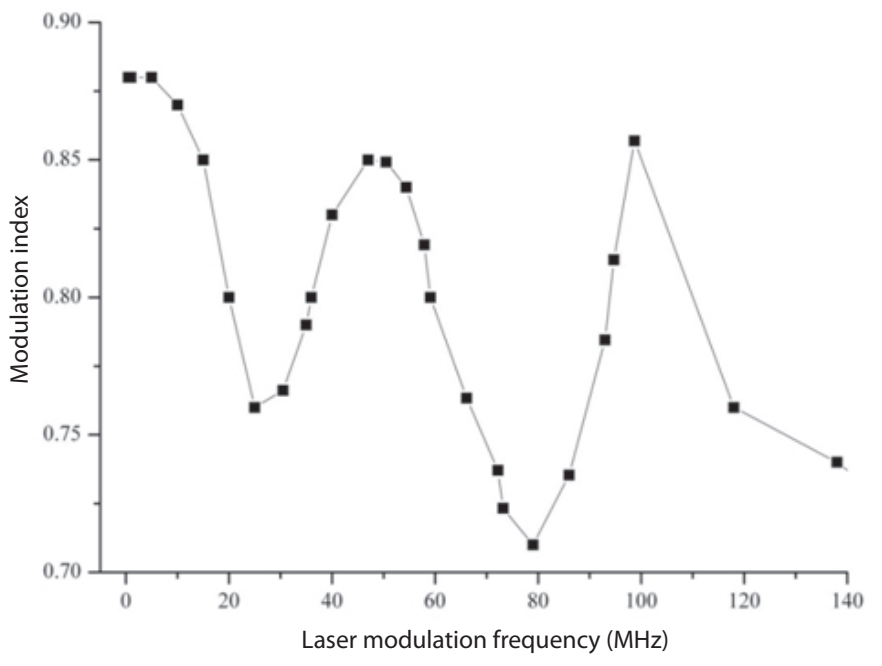

FIG. 3 Experimental determination of the laser modulation index vs. modulation frequency.

lated part of the backscattered signal vanishes for $f_{m} \gg f_{c}$ as the time that a photon travels without suffering a collision greatly exceeds the oscillation period of the laser's intensity. It is worthwhile to note that this is a typical result, rooted in the dissipation-fluctuation theorem [10] for physical systems subject to a periodical excitation and with a dissipative term depending only on one parameter, like for instance the susceptibility of Drude electrons in an electromagnetic field [11].

For a coherent detecting system, sensitive only to the modulated part of the signal operation in the $f_{m} \gg f_{c}$ regime ensures a considerable suppression of the optical noise coming from photons backscattered by water. The advantage of working in this regime, in a 3D imaging application, stems from the substantial independence from $f_{m}$ of the target signal amplitude.

\section{EXPERIMENTAL APPARATUS}

The launching stage of the AM laser 3D imager (see Figure 2), specifically set up to study the optical noise dependence on the RF modulation, consisted of a diode laser (Laser Technologies, Blue Photon) emitting CW radiation at $405 \mathrm{~nm}$ with power of $50 \mathrm{~mW}$. The laser radiation, delivered by a singlemode optical fiber with a core diameter of $4 \mu \mathrm{m}$, is collimated and amplitude modulated up to $200 \mathrm{MHz}$ with an analog signal provided by a lock-in amplifier (Standford Research SR844), which also measures the intensity and the phase difference, with respect to a reference, of the water backscattered radiation. The latter is collected by a short-focal-length lens of radius $r_{0}=2.5 \mathrm{~cm}$, focused and conveyed onto a fast photomultiplier detector (Hamamatsu H5783), forming a receiving optical stage with an angular field of view of $\theta_{f o v}=0.117 \mathrm{rad}$ (semiangle). A bistatic optical layout with a spatial separation of $r_{\text {rec }}=0.2 \mathrm{~m}$ between the light source and the receiver was adopted. The AM laser beam could freely propagate inside a black-walled $25 \mathrm{~m}$-long test tank, equipped with an antireflection coated entrance optical window. Signal contribution originating from unwanted reflections at the bottom wall of the tank was suppressed by means of a beam dump. During the experiments, the water extinction coefficient was varied by adding calibrated quantities of skim milk and measured by using a PerkinElmer Lambda $25 \mathrm{UV} /$ vis spectrometer in order to estimate the water turbidity.

\section{EXPERIMENTAL RESULTS}

In order to experimentally prove the low-pass filter dependence of the water backscattered power when the excitation comes from an AM laser source, measurements were performed with the test tank initially filled with tap-water $\left(k=0.5 \mathrm{~m}^{-1}\right)$. The extinction coefficient was subsequently increased up to $2.1 \mathrm{~m}^{-1}$ by adding controlled quantities of skimmed milk. From an experimental point of view, a major concern arises from the dependence of $m$ on $f_{m}$, that was then measured, as shown in Figure 3.

Up to the investigated frequency the modulation index shows an oscillating behavior, already observed in other AM systems [12] and likely due to a frequency dependent laser impedance, with a maximum value of 0.88 at $0.5 \mathrm{MHz}$ and minimum of 0.71 around $80 \mathrm{MHz}$. For frequencies larger than $140 \mathrm{MHz}, m$ falls rapidly to values lower than 0.5 making the system not reliable for quantitative measurements.

The results of three experiments at different values of $k$ are reported in Figure 4, where the experimental data are normalized to $m$. The experimental findings confirm the expected low-pass filter dependence and enable to verify that the width of the pass-band region is an increasing function of $k$. Nevertheless, it must be highlighted that the Butterworth's lowpass filter dependence, deriving from the simplified model described in Section 2, does not properly fit the experimental data, as the simulation for $k=0.5 \mathrm{~m}^{-1}$ reported in Figure 4 shows. Similar deviations can be also found for the other two experimental curves. This occurs because our experimental test bed significantly deviates from the ideal system assumed to find out Eq. (2), where heavy assumptions were made to simplify the treatment.

Adopting the more appropriate radiative transfer formalism to account for the bistatic layout and the finite value of the FOV introduces a new whole level of complexity into the theoretical treatment. Nevertheless, the here reported experimental data support our more accurate theoretical findings [9], resulting in an analytic expression in integral form for the re- 


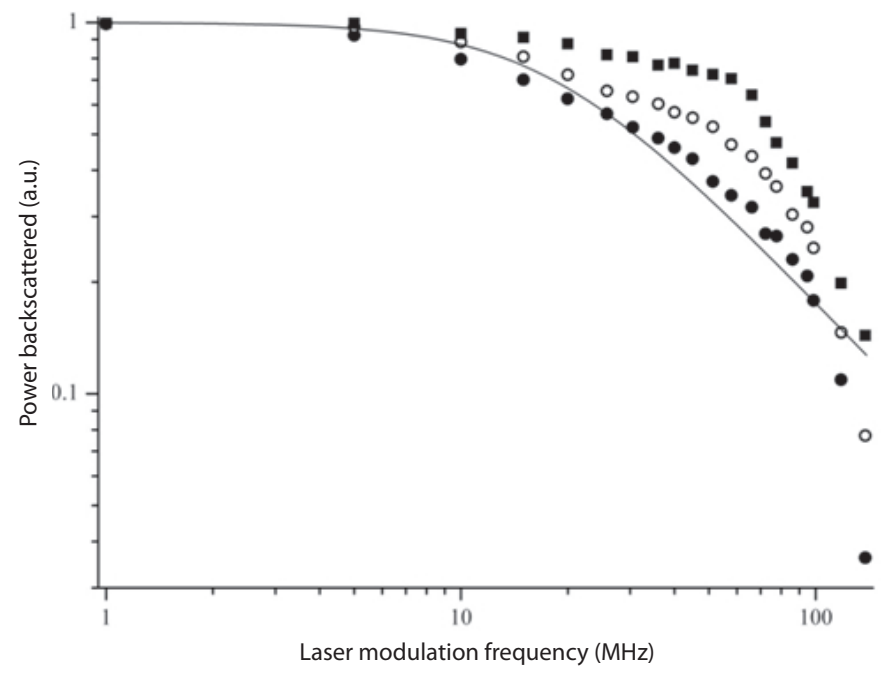

FIG. 4 Normalized backscattered power vs. laser modulation frequency measured for

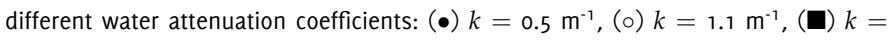
$2.1 \mathrm{~m}^{-1}$. The continuous curve is the plot of Butterworth's filter function in Eq. (2) for $k=0.5 \mathrm{~m}^{-1}$.

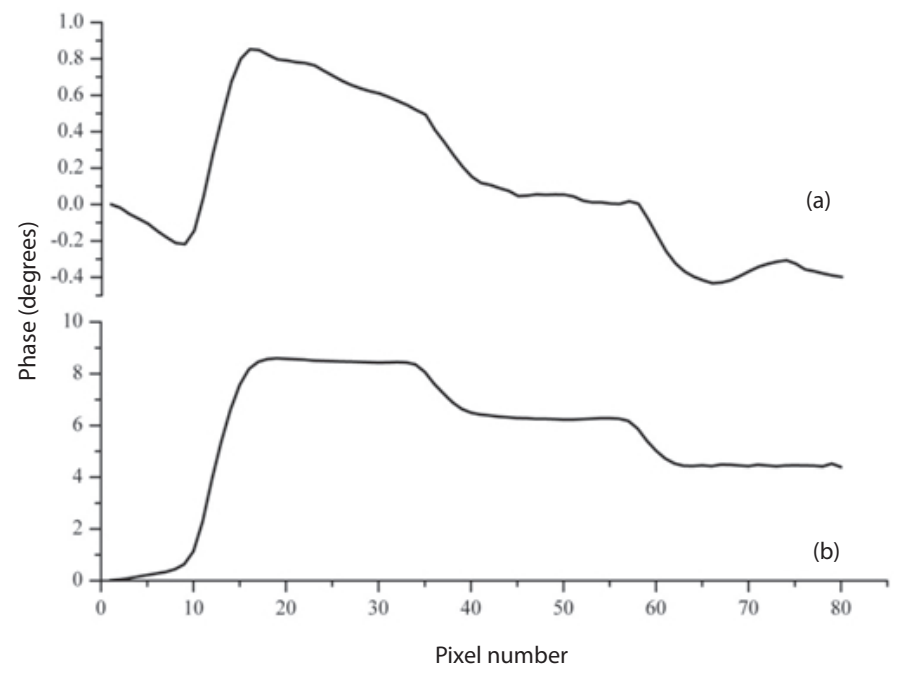

FIG. 5 Linear scan of the target profile for (a) $f_{m}=10 \mathrm{MHz}$ and (b) $f_{m}=85 \mathrm{MHz}$.

ceived backscattered power, where both water optical parameters and system factors, like $r_{r e c}, \theta_{\text {fov }}$ and $r_{0}$, compete to shape the low-pass filter function. However, the experimental conditions did not enable a direct determination of all the parameters entering the theoretical model in [9], thus not allowing at this stage a numerical simulation of the data in Figure 4, though a fitting procedure reproduces them quite well and with reasonable values for the unknown parameters.

After experimentally demonstrating the efficiency of optical noise suppression by means of modulation frequency control, the next experimental step was to verify an enhancement in target visualization when the device operates in the regime $f_{m} \gg f_{c}$. For this purpose we used as target a dark-graypainted, sanded, mostly diffusive, metallic calibrated ladder with $1 \mathrm{~cm}$ high steps, apart from the first step whose height was $4 \mathrm{~cm}$. The ladder was immersed at $3.7 \mathrm{~m}$ from the optical window of the test tank and its $8 \mathrm{~cm}$ long horizontal profile scanned in 80 pixels. The water attenuation coefficient was $0.5 \mathrm{~m}^{-1}$ resulting in a $f_{c}=17.9 \mathrm{MHz}$. The results of two dif- ferent scans at $f_{c}>f_{m}=10 \mathrm{MHz}$ and $f_{c}<f_{m}=85 \mathrm{MHz}$ are reported in Figure 5.

Despite for $f_{m}=85 \mathrm{MHz}$ the laser is operating with $m \sim 0.72$, the corresponding line profile reproduces with more accuracy the target shape than that recorded at $f_{m}=10 \mathrm{MHz}$ when $m \sim 0.87$. Since both the target signal $S$ and the optical noise $N$ scale linearly with $m$, the improvement in the image quality resulting in a better target profile reconstruction is rooted in the enhancement of the signaltonoise ratio $S / N$ due to the experimentally demonstrated low-pass filter dependence of $N$ on $f_{m}$. In both the recorded profiles, the smoothness of step-tostep jumps is a consequence of poor laser focalization (beam spot diameter on the target $0.5 \mathrm{~cm}$ ).

It must be noted that a complete extinction of the optical noise can be obtained only for $f_{m} / f_{c} \gg 10$ as Figure 1 shows, a condition out of the operating range of our equipment. This means that a residual optical noise contribution is still present at $f_{m}=85 \mathrm{MHz}$, as suggested by a quantitative analysis of the scanned linear profile. In fact, for the target signal, the measured phase $\phi$ depends linearly on target range $D$ through the relation $\phi=4 \pi n f_{m} D / c$. By assuming $n=1.344$, experimental data give $(\Delta \phi)_{\exp }=2.3^{\circ}$ for the $1 \mathrm{~cm}$ steps, a value lower than the theoretical prediction $(\Delta \phi)_{\text {theo }}=2.73^{\circ}$. The observed reduction of the measured range with respect to the real one is a property expected in our device when the optical noise contributes non negligibly to the detected signal. In fact the measured phase of a target at distance $\mathrm{D}$ is given by $\phi_{\exp }=$ $\tan ^{-1}\left(I_{q}^{t}+I_{q}^{n}\right) /\left(I_{p}^{t}+I_{p}^{n}\right)$ where $I_{q}$ and $I_{p}$ are the quadrature and in-phase components of the signal contribution from target $(t)$ and from noise $(r)$ respectively [13]. By using for the noise contribution the results obtained in Eq. (2) and for the target $I_{p}^{t}=I \cos \left(4 \pi n f_{m} D / c\right)$ and $I_{q}^{t}=I \sin \left(4 \pi n f_{m} D / c\right)$ it is possible to demonstrate that $(\Delta \phi)_{\exp }<(\Delta \phi)_{\text {theo }}$ for $I_{p}^{n}, I_{q}^{n} \neq 0$.

\section{SUMMARY}

The availability of a $25 \mathrm{~m}$-long test tank allowed us to experimentally investigate the low-pass-filter frequency dependence of the optical noise detected in an AM laser system aimed at $3 \mathrm{D}$ imaging of immersed target. The deviation from the Butterworth filter shape was expected on the basis of ongoing theoretical studies. In addition, improvements in the target profile reconstruction was demonstrated for the device operating in the stop-band frequency region. As far as we know, this is the first experimental demonstration that the phase measurement accuracy of an AM laser 3D imager can be enhanced just by adopting a modulation technique above the cut-off frequency for the rejection of optical noise. Advances in the theoretical studies, together with improvements in our experimental set-up, are in progress for providing a theoretical and experimental reliable estimation of the $f_{c}\left(k, r_{0}, r_{r e c}, \theta_{f o v}\right)$ function. At the same time, these investigations are also expected to shed light on the role played by the in-phase and quadrature components of the optical noise, just sketched here, and to ascertain the advantages of a 3D imaging system based on demodulated detection. 


\section{ACKNOWLEDGMENTS}

Authors acknowledge M. Sighicelli, A. Lai and L. Bartolini for their contribution. This work was funded by BLU-Archeosys project.

\section{References}

[1] D. M. Kocak, F. R. Dalgleish, F. M. Caimi, and Y. Y. Schechner, "A focus on recent development and trend in underwater imaging" Mar. Technol. Soc. J. 42, 52-67 (2008).

[2] J. S. Jaffe, K. D. Moore, J. McLean, and M. P. Strand, “Underwater optical imaging: status and prospects" Oceanography 14, 64-75 (2001).

[3] L. Bartolini, L. De Dominicis, M. Ferri de Collibus, G. Fornetti, M. Guarneri, E. Paglia, C. Poggi, and R. Ricci, "Underwater threedimensional imaging with an amplitude modulated laser radar at 405 nm" Appl. Opt. 44, 7130-7135 (2005).

[4] F. Pellen, X. Intes, P. Olivard, Y. Guern, J. Cariou, and J. Lotrian, "Determination of sea-water cut-off frequency by backscattering transfer function measurement" J. Phys. D Appl. Phys. 33, 349-354 (2000).

[5] L. Mullen, A. Vieira, P. Herczfeld, and M. Contarino, “Application of RADAR technology to aerial LIDAR systems for enhancement of shallow underwater target detection" IEEE T. Microw. Theory 43, 2370-2377 (1995).
[6] F. Pellen, P. Olivard, Y. Guern, J. Cariou, and J. Lotrian, "Radio frequency modulation on an optical carrier for target detection enhancement in sea-water" J. Phys. D Appl. Phys. 34, 1122-1130 (2001).

[7] L. Mullen, A. Laux, B. Concannon, E. P. Zege, I. L. Katsev, and A. S. Prikhach, "Amplitude-modulated laser imager" Appl. 0pt. 43 , 3874-3892 (2004).

[8] I. L. Katsev, E. P. Zege, A. S. Prikhach, and I. N. Polonsky, “Efficient technique to determine backscattered light power for various atmospheric and oceanic sounding and imaging systems" J. Opt. Soc. Am. A 14, 1338-1346 (1997).

[9] R. Ricci, M. Francucci, L. De Dominicis, M. Ferri de Collibus, G. Fornetti, M. Guarneri, M. Nuvoli, E. Paglia, and L. Bartolini, "Techniques for effective optical noise rejection in amplitude-modulated laser optical radars for underwater three-dimensional imaging" submitted to EURASIP J. Adv. Sig. Pr. (2009).

[10] G. F. Giuliani, and G. Vignale, Quantum theory of the electron liquid (Cambridge University Press, Cambridge, 2005).

[11] M. Dresse, and G. Grüner, Electrodynamics of solids (Cambridge University Press, Cambridge, 2002).

[12] J. Peng, L. Wei, and Q. Wang, "Broadband amplitude modulated laser source with high output power for FM/cw LIDAR transmitter" J. Russ. Laser Res. 30, 253-259 (2009).

[13] M. 0. Sonnaillon, and F. J. Bonetto, "Lock-in amplifier error prediction and correction in frequency sweep measurements" Rev. Sci. Instrum. 78, 014701 (2007). 